Article

\title{
Probabilistic Interpretation of Solutions of Linear Ultraparabolic Equations
}

\author{
Michael D. Marcozzi \\ Department of Mathematical Sciences, University of Nevada Las Vegas, Las Vegas, NV 89154-4020, USA; \\ michael.marcozzi@unlv.edu
}

Received: 1 November 2018; Accepted: 21 November 2018; Published: 27 November 2018

\begin{abstract}
We demonstrate the existence, uniqueness and Galerkin approximatation of linear ultraparabolic terminal value/infinite-horizon problems on unbounded spatial domains. Furthermore, we provide a probabilistic interpretation of the solution in terms of the expectation of an associated ultradiffusion process.
\end{abstract}

Keywords: ultraparabolic equation; ultradiffusion process; probabilistic representation; mathematical finance

\section{Introduction}

The connection between parabolic equations and diffusion processes is well understood; the same cannot be said for ultraparabolic equations and ultradiffusion processes. Until recently, theoretical results have been fairly limited relative to the existence and uniqueness of solutions to ultraparabolic equations, deriving from two methodologies. In one, the analysis is affected along the characteristic of the first-order temporal operator, requiring that the speed of propagation varies only spatially. Such an approach was developed by Piskunov [1] in the classical case and extended by Lions [2] to the generalized sense. The second approach is based on the method of fundamental solutions and was implemented by Il'in [3] for the classical Cauchy problem and extended to more general domains via convolution by Vladimirov and Drožžinov [4], albeit at the expense of necessitating constant coefficients in the operator. Recently, however, using energic techniques Marcozzi [5] has established the well-posedness and Galerkin approximation of the generalized solution (strong and weak) to the terminal value problem for square integrable data on bounded temporal and spatial domains. We extend here the results of [5] to linear ultraparabolic terminal value/infinite-horizon temporal problems posed on unbounded spatial domains. We then provide a probabilistic interpretation of the solution in terms of the expectation of an associated ultradiffusion process.

Historically, the connection between the expectation of ultradiffusion processess and the solution to ultradiffusion equations arose from the work of Kolmogorov [6,7] and Uhlenbeck and Ornstein [8] in relation to Brownian motion in phase space-the same with respect to Chandrasekhar [9] in the context of boundary layers and Marshak [10] relative to the Bolzmann equation. A contemporary example may be found in the formulation of so-called Asian options from mathematical finance (cf. [11]), which obtains theoretical context with the present results. The paper is organized as follows. In Section 2, we consider deterministic aspects of the problem, while, in Section 3, the probabilistic interpretation is presented. Appendix A introduces certain regularity results, which, while essential for the analysis, are too extensive to prove in full. In Appendix B, we show formally that the ultraparabolic/ultradiffusion association is locally that of a parameterized parabolic/diffusion. 


\section{Approximation Solvability}

We consider here the existence, uniqueness and approximation of the terminal value/infinite horizon problem on unbounded spatial domains for the linear ultraparabolic equations. To this end, let $\widetilde{\mathcal{O}}_{T, \vartheta}=(0, T) \times(0, \infty), \widetilde{\mathcal{O}}_{\vartheta, x}=(0, \infty) \times(-\infty, \infty), \widetilde{\mathcal{O}}_{T, x}=(0, T) \times(-\infty, \infty)$, and finally $\widetilde{\mathcal{Q}}=(0, T) \times(0, \infty) \times(-\infty, \infty)$, for some finite $T>0$. The functional setting will be the weighted Sobolev spaces defined as follows. Spatially, we let

$$
m_{\mu}(x)=e^{-\mu|x|}
$$

such that

$$
H_{\mu}=\left\{v(x) \mid m_{\mu}(x) v(x) \in L_{2}(\mathbb{R})\right\}
$$

and

$$
V_{\mu}=\left\{v \in H_{\mu} \mid \partial v(x) / \partial x \in H_{\mu}\right\}
$$

with their respective norms

$$
|v|_{\mu}=\left\{\int_{\mathbb{R}}\left[m_{\mu}(x) v(x)\right]^{2} d x\right\}^{1 / 2}
$$

for all $v \in H_{\mu}$, and

$$
\|v\|_{\mu}=\left\{|v|_{\mu}^{2}+|\partial v / \partial x|_{\mu}^{2}\right\}^{1 / 2},
$$

for all $v \in V_{\mu}$. The relation " $V_{\mu} \subseteq H_{\mu} \subset V_{\mu}^{* \prime}$ constitutes an evolution triple.

Temporally, let $\boldsymbol{t}=(t, \vartheta)$ and

$$
n_{\gamma}(\boldsymbol{t})=e^{-\gamma \vartheta},
$$

such that

$$
\mathcal{X}_{\gamma, \mu}=L_{\gamma}^{2}\left(\widetilde{\mathcal{O}}_{T, \vartheta} ; V_{\mu}\right)=\left\{u(\boldsymbol{t}) \mid n_{\gamma}(\boldsymbol{t}) u(\boldsymbol{t}) \in L_{2}\left(\widetilde{\mathcal{O}}_{T, \vartheta} ; V_{\mu}\right)\right\},
$$

which we equip with the norm

$$
\|u\|_{\gamma, \mu}=\|u\|_{\mathcal{X}_{\gamma, \mu}}=\left\{\int_{\widetilde{\mathcal{O}}_{T, \vartheta}}\left\|n_{\gamma}(\boldsymbol{t}) u(\boldsymbol{t})\right\|_{\mu}^{2} d \mathcal{O}\right\}^{1 / 2},
$$

for all $u \in \mathcal{X}_{\gamma, \mu}$. We associate with $\mathcal{X}_{\gamma, \mu}$ the dual space

$$
\mathcal{X}_{\gamma, \mu}^{*}=L_{\gamma}^{2}\left(\mathcal{O}_{t, \vartheta} ; V_{\mu}^{*}\right)
$$

and the norm $\left\|u^{*}\right\|_{\mathcal{X}_{\gamma, \mu}^{*}}$ for all $u^{*} \in \mathcal{X}_{\gamma, \mu}$. In addition, let

$$
W_{\gamma, \mu}=W_{\gamma}^{1}\left(\mathcal{O}_{t, \vartheta} ; V_{\mu}, H_{\mu}\right)=\left\{u \in \mathcal{X}_{\gamma, \mu}: \nabla_{t}(u) \in \mathcal{X}_{\gamma, \mu}^{*} \times \mathcal{X}_{\gamma, \mu}^{*}\right\},
$$

where $\nabla_{t}(u)=(\partial u / \partial t, \partial u / \partial \vartheta)$, which we associate with the norm

$$
\|u\|_{W_{\gamma, \mu}}=\left(\|u\|_{\mathcal{X}_{\gamma, \mu}}^{2}+\|\partial u / \partial t\|_{\mathcal{X}_{\gamma, \mu}^{*}}^{2}+\|\partial u / \partial \vartheta\|_{\mathcal{X}_{\gamma, \mu}^{*}}^{2}\right)^{2},
$$

for all $u \in W_{\gamma, \mu}$. Finally, we define

$$
L_{\gamma}^{2}\left((0, \infty) ; H_{\mu}\right)=\left\{u(\vartheta) \mid n_{\gamma}(\boldsymbol{t}) u(\boldsymbol{t}) \in L_{2}\left(\widetilde{\mathcal{O}}_{T, \vartheta} ; H_{\mu}\right)\right\} .
$$

We consider the $u$ ltraparabolic $t$-terminal value/infinite $\vartheta$-horizon problem for $u \in W_{\gamma, \mu}$ satisfying the evolutionary equation 


$$
-\frac{\partial u}{\partial t}-\frac{\partial(b u)}{\partial \vartheta}+A(\boldsymbol{t}) u=f \text { a.e. on } \widetilde{\mathcal{Q}},
$$

subject to the terminal condition

$$
u(T, \vartheta, x)=v(\vartheta, x) \text { a.e. on } \widetilde{\mathcal{O}}_{\vartheta, x}
$$

where

$$
A(\boldsymbol{t}) u=-\frac{\partial}{\partial x}\left(a_{2} \frac{\partial u}{\partial x}\right)+a_{1} \frac{\partial u}{\partial x}+a_{0} u
$$

for given

$$
\begin{gathered}
a_{0}, a_{1}, a_{2} \in L_{\infty}(\widetilde{\mathcal{Q}}), b \in C^{1}(\widetilde{\widetilde{\mathcal{Q}}}) \\
0<\underline{b} \leq b(\boldsymbol{t}, x) \leq 2 \bar{b}, \partial b / \partial \vartheta \text { bounded } \\
v \in L_{\gamma}^{2}\left((0, \infty) ; H_{\mu}\right), \\
\quad f \in \mathcal{X}_{\gamma, \mu}^{*}, \\
0<\alpha \leq a_{2} \text { and } 0<\beta \leq a_{0},
\end{gathered}
$$

for some sufficiently large $\beta$.

The generalized problem associated with (1)-(2) is: supposing (3)-(7), find $u \in W_{\gamma, \mu}$ satisfying

$$
-\frac{\partial}{\partial t}(u(\boldsymbol{t}) \mid v)_{H_{\mu}}-\frac{\partial}{\partial \vartheta}(b(\boldsymbol{t}) u(\boldsymbol{t}) \mid v)_{H_{\mu}}+a_{\mu}(\boldsymbol{t} ; u(\boldsymbol{t}), v)=\langle f(\boldsymbol{t}), v\rangle_{V_{\mu}},
$$

for almost all $t \in \widetilde{\mathcal{O}}_{T, \vartheta}$, such that

$$
u\left(\boldsymbol{T}_{\vartheta}\right)=v(\vartheta) \text { on } \widetilde{\mathcal{O}}_{\vartheta, x},
$$

where $(u \mid v)_{X}$ is the scalar product canonically defined on the Hilbert space $X,\langle f(\boldsymbol{t}), v\rangle_{V_{\mu}}$ denotes the value of the linear functional $f(\boldsymbol{t}) \in V_{\mu}^{*}$ at $v \in V_{\mu}, \boldsymbol{T}_{\vartheta}=(T, \vartheta)$ and

$$
a_{\mu}(\boldsymbol{t} ; u, v)=\int_{\mathbb{R}} a_{2} \frac{\partial u}{\partial x} \frac{\partial v}{\partial x} m_{\mu}^{2} d x+\int_{\mathbb{R}} a_{1} \frac{\partial u}{\partial x} v m_{\mu}^{2} d x+\int_{\mathbb{R}} a_{0} u v m_{\mu}^{2} d x,
$$

for all $u, v \in V_{\mu}$ and $t \in \widetilde{\mathcal{O}}_{T, \vartheta}$. In Equation (8), the expressions $\partial / \partial t$ and $\partial / \partial \vartheta$ denote generalized derivatives on $\widetilde{\mathcal{O}}_{T, \vartheta}$; that is, Equation (8) means explicitly

$$
\begin{gathered}
\int_{\widetilde{\mathcal{O}}_{T, \vartheta}}(u(\boldsymbol{t}) \mid v)_{H_{\mu}} \frac{\partial}{\partial t} \varphi(\boldsymbol{t}) n_{\gamma}^{2} d \mathcal{O}+\int_{\widetilde{\mathcal{O}}_{T, \vartheta}}(b(\boldsymbol{t}) u(\boldsymbol{t}) \mid v)_{H_{\mu}} \frac{\partial}{\partial \vartheta} \varphi(\boldsymbol{t}) n_{\gamma}^{2} d \mathcal{O} \\
+\int_{\widetilde{\mathcal{O}}_{T, \vartheta}} a(\boldsymbol{t} ; u(\boldsymbol{t}), v) \varphi(\boldsymbol{t}) d \mathcal{O}=\int_{\widetilde{\mathcal{O}}_{T, \vartheta}}\langle f(\boldsymbol{t}), v\rangle_{V_{\mu}} \varphi(\boldsymbol{t}) n_{\gamma}^{2} d \mathcal{O},
\end{gathered}
$$

for all test functions $\varphi \in C_{0}^{\infty}\left(\widetilde{\mathcal{O}}_{T, \vartheta}\right)$.

For $\boldsymbol{t} \in \widetilde{\mathcal{O}}_{T, \vartheta}$, the mapping $a_{\mu}(\boldsymbol{t}): V_{\mu} \times V_{\mu}$ is bilinear and bounded; we likewise assume that $a_{\mu}(\boldsymbol{t})$ is strongly positive;

$$
c\|u(\boldsymbol{t})\|_{\mu}^{2} \leq a_{\mu}(\boldsymbol{t} ; u(\boldsymbol{t}), u(\boldsymbol{t})) .
$$

Remark 1. We note that Equations (1)-(2) is an infinite horizon problem in $\vartheta$. That is, the far-field behavior of $\vartheta$ is implicitly defined relative to the weight $\gamma$.

Remark 2. In general, the validity of (11) will be problem dependent, predicated upon the spatial asymptotic behavior of $u$.

For $\boldsymbol{t} \in \widetilde{\mathcal{O}}_{T, \vartheta}$, we define the operator $A_{\mu}(\boldsymbol{t}): V_{\mu} \rightarrow V_{\mu}^{*}$ such that 


$$
\left\langle A_{\mu}(\boldsymbol{t}) u, v\right\rangle_{V_{\mu}}=a_{\mu}(\boldsymbol{t} ; u, v)
$$

from which it follows that $A_{\mu}(\boldsymbol{t})$ is linear, continuous, and strongly monotone by (11). In particular, we have

$$
\|A(\boldsymbol{t}) u\|_{\mathcal{X}_{\gamma, \mu}^{*}} \leq C\|u\|_{\mathcal{X}_{\gamma, \mu}}
$$

and

$$
c\|u\|_{\mu}^{2} \leq\langle A(\boldsymbol{t}) u, u\rangle_{V_{\mu}},
$$

for all $u \in V_{\mu}$ and $\boldsymbol{t} \in \widetilde{\mathcal{O}}_{T, \vartheta}$.

Lemma 1. Given (3)-(7), the formulations (1)-(2) and (8)-(9) are equivalent.

Proof of Lemma 1. By integration by parts and the density of test functions in $V_{\mu}$, we have

$$
\frac{\partial}{\partial t}(u(\boldsymbol{t}) \mid v)=\left\langle\frac{\partial u(\boldsymbol{t})}{\partial t}, v\right\rangle_{V_{\mu}} \text { and } \frac{\partial}{\partial \vartheta}(u(\boldsymbol{t}) \mid v)=\left\langle\frac{\partial u(\boldsymbol{t})}{\partial \vartheta}, v\right\rangle_{V_{\mu}}
$$

for all $v \in V_{\mu}$ and almost all $t \in \widetilde{\mathcal{O}}_{T, \vartheta}$. From (8), (12) and (15), we deduce that

$$
\left\langle-\frac{\partial u(\boldsymbol{t})}{\partial t}-\frac{\partial(b(\boldsymbol{t}) u(\boldsymbol{t}))}{\partial \vartheta}+A(\boldsymbol{t}) u(\boldsymbol{t})-f(\boldsymbol{t}), v\right\rangle_{V_{\mu}}=0
$$

for all $v \in V_{\mu}$ and almost all $t \in \mathcal{O}_{T, \Theta}$, in which case (1) follows. The converse derives from (1) and $u \in W_{\gamma, \mu}$, which imply (8).

Proposition 1. Uniqueness. We suppose (3)-(7) and (11); let $0<\gamma<c$. Then, there exists at most one solution to (8)-(9).

Proof of Proposition 1. We consider (8)-(9) with $f=0$ and $v=0$; setting $v=u$ in (8), we obtain

$$
-\frac{1}{2} \frac{\partial}{\partial t}|u(\boldsymbol{t})|_{\mu}^{2}-\frac{1}{2} \frac{\partial}{\partial \vartheta}|\sqrt{b(\boldsymbol{t})} u(\boldsymbol{t})|_{\mu}^{2}+a_{\mu}(\boldsymbol{t} ; u(\boldsymbol{t}), u(\boldsymbol{t}))=0
$$

or

$$
2 c\|u(t)\|_{\mu}^{2} \leq \frac{\partial}{\partial t}|u(\boldsymbol{t})|_{\mu}^{2}+\frac{\partial}{\partial \vartheta}|\sqrt{b(t)} u(t)|_{\mu}^{2},
$$

from (11), in which case

$$
0 \leq \frac{\partial}{\partial t}\left(e^{-2 c \vartheta}|u(\boldsymbol{t})|_{\mu}^{2}\right)+\frac{\partial}{\partial \vartheta}\left(e^{-2 c \vartheta}|\sqrt{b(\boldsymbol{t})} u(\boldsymbol{t})|_{\mu}^{2}\right) .
$$

Integrating over the domain $(0, T) \times(0, \Theta)$, for some $\Theta>0$, and applying Green's Theorem, it follows that

$$
\int_{0}^{\Theta} e^{-2 c \vartheta}|u(0, \vartheta)|_{\mu}^{2} d \vartheta+\int_{0}^{T}|\sqrt{b(t, 0)} u(t, 0)|_{\mu}^{2} d t \leq \int_{0}^{T} e^{-2 c \Theta}|\sqrt{b(t, \Theta)} u(t, \Theta)|_{\mu}^{2} d t
$$

and so

$$
0<\tilde{c} \leq e^{-2 c \Theta}|u(t, \Theta)|_{\mu}^{2}
$$

However,

$$
\tilde{c} e^{-2(c-\gamma) \Theta} \leq e^{-2 c \Theta}|u(t, \Theta)|_{\mu}^{2}
$$


is not summable on $(0, \infty)$, which contradicts the condition $u \in \mathcal{X}_{\gamma, \mu}$, from which it follows that $u=0$.

We consider the regularization of (1)-(2) to domains of finite extent. To this end, it suffices for $v$ to have an extension to, or to be of compact support in, $\widetilde{\mathcal{Q}}$. Without loss of generality, we may assume that $v=0$. For $m \in \mathbb{N}$, let

$$
\begin{gathered}
\mathcal{Q}_{m}=(0, T) \times(0, m) \times(-m, m), \\
f_{m}=f \text { on } \mathcal{Q}_{m}, \\
V_{m}=H_{0}^{1}(-m, m), \\
H_{m}=L_{2}(-m, m), \\
\mathcal{X}_{m}=L_{2}\left((0, T) \times(0, m) ; V_{m}\right), \\
\mathcal{X}_{m}^{*}=L_{2}\left((0, T) \times(0, m) ; V_{m}^{*}\right),
\end{gathered}
$$

and

$$
W_{m}=\left\{u_{m} \in \mathcal{X}_{m} \mid \nabla_{t}\left(u_{m}\right) \in \mathcal{X}_{m}^{*} \times \mathcal{X}_{m}^{*}\right\} .
$$

There exists a unique $u_{m} \in W_{m}$ satisfying the ultraparabolic terminal value problem (cf. [5])

$$
-\frac{\partial u_{m}}{\partial t}-\frac{\partial\left(b u_{m}\right)}{\partial \vartheta}+A(t, \vartheta) u_{m}=f_{m} \text { a.e. on } \mathcal{Q}_{m}
$$

subject to the terminal conditions

$$
\begin{aligned}
& u(T, \vartheta, x)=0 \text { a.e. on }(0, m) \times(-m, m), \\
& u(t, m, x)=0 \text { a.e. on }(0, T) \times(-m, m),
\end{aligned}
$$

and boundary conditions

$$
u(t, \vartheta,-m)=u(t, \vartheta,-m)=0 \text { a.e. on }(0, T) \times(0, m) .
$$

We denote by $\widetilde{u}_{m}$ the extension of $u_{m}$ by zero to the compliment of $\mathcal{Q}_{m}$.

Lemma 2. We suppose (3)-(7), (11),

$$
\nabla_{t}\left(a_{i}\right) \in L_{\infty}(\widetilde{\mathcal{Q}}) \times L_{\infty}(\widetilde{\mathcal{Q}}),
$$

$0<\gamma<c / \bar{b}$ and $f \in L_{\gamma}^{2}\left(\widetilde{\mathcal{O}}_{t, \vartheta} ; H_{\mu}\right)$; then,

$$
\left\|\tilde{u}_{m}\right\|_{\gamma, \mu} \leq C,
$$

for all $m \in \mathbb{N}$.

Proof of Lemma 2. Taking the inner product of (16) with $\widetilde{u}_{m}$, we have

$$
-\left(\frac{\partial \widetilde{u}_{m}}{\partial t}, \widetilde{u}_{m}\right)_{\mu}-\left(\frac{\partial b \widetilde{u}_{m}}{\partial \vartheta}, \widetilde{u}_{m}\right)_{\mu}+a_{\mu}\left(\boldsymbol{t} ; \widetilde{u}_{m}, \widetilde{u}_{m}\right)=\left(f_{m}, \widetilde{u}_{m}\right)_{\mu}
$$

or

$$
-e^{-2 \gamma \vartheta} \frac{1}{2} \frac{\partial}{\partial t}\left|\widetilde{u}_{m}\right|_{\mu}^{2}-e^{-2 \gamma \vartheta} \frac{1}{2} \frac{\partial}{\partial \vartheta}\left|\sqrt{b} \widetilde{u}_{m}\right|_{\mu}^{2}+c e^{-2 \gamma \vartheta}\left\|\widetilde{u}_{m}\right\|_{\mu}^{2} \leq e^{-2 \gamma \vartheta}\left(f_{m}, \widetilde{u}_{m}\right)_{\mu} .
$$


Integrating the above over $(0, T) \times(\vartheta, m)$, it follows that

$$
\begin{gathered}
-\int_{0}^{T} \int_{\vartheta}^{m} e^{-2 \gamma \vartheta} \frac{1}{2} \frac{\partial}{\partial t}\left|\widetilde{u}_{m}\right|_{\mu}^{2}-\int_{0}^{T} \int_{\vartheta}^{m} e^{-2 \gamma \vartheta} \frac{1}{2} \frac{\partial}{\partial \vartheta}\left|\sqrt{b} \widetilde{u}_{m}\right|_{\mu}^{2} \\
+c \int_{0}^{T} \int_{\vartheta}^{m} e^{-2 \gamma \vartheta}\left\|\widetilde{u}_{m}\right\|_{\mu}^{2} \leq \int_{0}^{T} \int_{\vartheta}^{m} e^{-2 \gamma \vartheta}\left(f_{m}, \widetilde{u}_{m}\right)_{\mu}
\end{gathered}
$$

or

$$
\begin{gathered}
-\left\{\int_{0}^{T} \int_{\vartheta}^{m} \frac{\partial}{\partial t}\left[e^{-2 \gamma \vartheta}\left|\widetilde{u}_{m}\right|_{\mu}^{2}\right]+\int_{0}^{T} \int_{\vartheta}^{m} \frac{\partial}{\partial \vartheta}\left[e^{-2 \gamma \vartheta}\left|\sqrt{b} \widetilde{u}_{m}\right|_{\mu}^{2}\right]\right\} \\
-\int_{0}^{T} \int_{\vartheta}^{m} 2 \gamma e^{-2 \gamma \vartheta}\left|\sqrt{b} \widetilde{u}_{m}\right|_{\mu}^{2}+2 c \int_{0}^{T} \int_{\vartheta}^{m} e^{-2 \gamma \vartheta}\left\|\widetilde{u}_{m}\right\|_{\mu}^{2} \\
\leq 2 \int_{0}^{T} \int_{\vartheta}^{m} e^{-2 \gamma \vartheta}\left(f_{m}, \widetilde{u}_{m}\right)_{\mu}
\end{gathered}
$$

and so

$$
\begin{aligned}
\int_{\vartheta}^{m} e^{-2 \gamma \vartheta} & \left|\widetilde{u}_{m}(0, \vartheta)\right|_{\mu}^{2}+\int_{0}^{T} e^{-2 \gamma \vartheta}\left|\widetilde{u}_{m}(t, \vartheta)\right|_{\mu}^{2}-\int_{0}^{T} \int_{\vartheta}^{m} 2 \gamma e^{-2 \gamma \vartheta}\left|\sqrt{b} \widetilde{u}_{m}\right|_{\mu}^{2} \\
& +2 c \int_{0}^{T} \int_{\vartheta}^{m} e^{-2 \gamma \vartheta}\left\|\widetilde{u}_{m}\right\|_{\mu}^{2} \leq 2 \int_{0}^{T} \int_{\vartheta}^{m} e^{-2 \gamma \vartheta}\left(f_{m}, \widetilde{u}_{m}\right)_{\mu}
\end{aligned}
$$

in which case

$$
\begin{gathered}
2 c \int_{0}^{T} \int_{\vartheta}^{m} e^{-2 \gamma \vartheta}\left\|\widetilde{u}_{m}\right\|_{\mu}^{2}-\int_{0}^{T} \int_{\vartheta}^{m} 2 \gamma e^{-2 \gamma \vartheta}\left|\sqrt{b} \widetilde{u}_{m}\right|_{\mu}^{2} \\
\leq 2 \int_{0}^{T} \int_{\vartheta}^{m} e^{-2 \gamma \vartheta}\left(f_{m}, \widetilde{u}_{m}\right)_{\mu}
\end{gathered}
$$

or

$$
\begin{aligned}
& (c-\bar{b} \gamma) \int_{0}^{T} \int_{\vartheta}^{m} e^{-2 \gamma \vartheta}\left\|\widetilde{u}_{m}\right\|_{\mu}^{2} \leq \int_{0}^{T} \int_{\vartheta}^{m} e^{-2 \gamma \vartheta}\left(f_{m}, \widetilde{u}_{m}\right)_{\mu} \\
& \leq\left(\int_{0}^{T} \int_{\vartheta}^{m} e^{-2 \gamma \vartheta}\left\|\widetilde{f}_{m}\right\|_{\mu}^{2}\right)^{1 / 2}\left(\int_{0}^{T} \int_{\vartheta}^{m} e^{-2 \gamma \vartheta}\left\|\widetilde{u}_{m}\right\|_{\mu}^{2}\right)^{1 / 2}
\end{aligned}
$$

such that

$$
\int_{0}^{T} \int_{\vartheta}^{m} e^{-2 \gamma \vartheta}\left\|\widetilde{u}_{m}\right\|_{\mu}^{2} \leq C
$$

therefore,

$$
\left\|\tilde{u}_{m}\right\|_{\gamma, \mu} \leq C
$$

for all $m \in \mathbb{N}$.

We obtain a supplementary estimate on $\nabla_{t}\left(\widetilde{u}_{m}\right)$.

Lemma 3. We suppose (3)-(7), (11), (20), $0<\gamma<c / \bar{b}$ and $f \in L_{\gamma}^{2}\left(\mathcal{O}_{t, \vartheta} ; H_{\mu}\right)$, then

$$
\left\|\frac{\partial \widetilde{u}_{m}}{\partial t}\right\|_{\gamma, \mu} \leq C \text { and }\left\|\frac{\partial \widetilde{u}_{m}}{\partial \vartheta}\right\|_{\gamma, \mu} \leq C
$$

Proof of Lemma 3. We consider the parabolic regularization with respect to $\vartheta$ of (16)-(19). To this end, let $\mathcal{H}_{m}(0, \Theta)=L_{2}\left(0, \Theta ; H_{m}\right)$; we define the space of test functions on $(0, m) \times(-m, m)$ such that

$$
\mathcal{V}_{m}(0, m)=\left\{v \mid v, \frac{\partial v}{\partial x}, \frac{\partial v}{\partial \vartheta} \in L_{2}\left(0, m ; H_{m}\right), v(t, \vartheta,-m)=v(t, \vartheta, m)=v(t, m, x)=0\right\},
$$


in which case we obtain the evolution triple " $\mathcal{V}_{m}(0, m) \subset \mathcal{H}_{m}(0, m) \subset \mathcal{V}_{m}^{*}(0, m)$ ". We denote $\mathcal{H}_{m}=\mathcal{H}_{m}(0, m)$ and $\mathcal{V}_{m}=\mathcal{V}_{m}(0, m)$ for brevity and equip $\mathcal{V}_{m}$ with the norm

$$
\begin{gathered}
\|v\|_{\mathcal{V}_{m}}^{2}=\int_{(0, m)}\|v(\theta)\|_{H_{m}}^{2} d \theta+\int_{(0, m)}\|\partial v(\theta) / \partial x\|_{H_{m}}^{2} d \theta+\int_{(0, m)}\|\partial v(\theta) / \partial \theta\|_{H_{m}}^{2} d \theta \\
=\|v\|_{\mathcal{H}_{m}}^{2}+\|\partial v / \partial x\|_{\mathcal{H}_{m}}^{2}+\|\partial v / \partial \vartheta\|_{\mathcal{H}_{m}}^{2} .
\end{gathered}
$$

Let

$$
\mathcal{W}_{m}=\mathcal{W}_{m}\left(0, T ; \mathcal{V}_{m}, \mathcal{H}_{m}\right)=\left\{v \mid v \in \mathcal{X}_{m}, \frac{\partial v}{\partial t} \in \mathcal{X}_{m}^{*}, \frac{\partial v}{\partial \vartheta} \in \mathcal{X}_{m}^{*}, v(t, m, x)=0\right\}
$$

where $\mathcal{X}_{m}=L_{2}\left((0, m) \times(-m . m) ; V_{m}\right)$ and $\mathcal{X}_{m}^{*}=L_{2}\left((0, m) \times(-m . m) ; V_{m}^{*}\right)$, which we equip with the norm

$$
\|v\|_{\mathcal{W}_{m}}^{2}=\int_{0}^{T}\|v(\tau)\|_{\mathcal{V}_{m}}^{2} d \tau+\int_{0}^{T}\|\partial v(\tau) / \partial \tau\|_{\mathcal{X}_{m}^{*}}^{2} d \tau
$$

The perturbation problem associated with (16)-(19) is: for any $\epsilon>0$, we seek $u_{m}^{\epsilon}$ satisfying the parabolic equation

$$
-\frac{\partial u_{m}^{\epsilon}}{\partial t}-\epsilon \frac{\partial^{2} u_{m}^{\epsilon}}{\partial \vartheta^{2}}-\frac{\partial\left(b u_{m}^{\epsilon}\right)}{\partial \vartheta}+A(\boldsymbol{t}) u_{m}^{\epsilon}=f_{m} \text { a.e. on } \mathcal{Q},
$$

where $A(t)=A(t, \vartheta)$, subject to the terminal condition

$$
u_{m}^{\epsilon}(T, \vartheta, x)=v(\vartheta, x) \text { a.e. on }(0, m) \times(-m, m),
$$

and boundary conditions

$$
\begin{gathered}
u_{m}^{\epsilon}(t, \Theta, x)=0 \text { a.e. on }(0, T) \times(-m, m), \\
u^{\epsilon}(t, \vartheta,-m)=u^{\epsilon}(t, \vartheta, m)=0 \text { a.e. on }(0, t) \times(0, m), \\
\frac{\partial u_{m}^{\epsilon}}{\partial \vartheta}(t, m, x)=0 \text { a.e. on }(0, T) \times(-m, m) .
\end{gathered}
$$

The problem (23)-(27) is well-posed, noting in particular the necessity of the auxiliary boundary condition (27).

We denote by $\widetilde{u}_{m}^{e}$ the extension of $u_{m}^{\epsilon}$ by zero to the compliment of $\mathcal{Q}_{m}$. Taking the inner product of (23) with $-n_{\gamma}^{2} \partial \widetilde{u}_{m}^{\epsilon} / \partial t$, it follows that

$$
\int_{(0, m)}\left(\frac{\partial \widetilde{u}_{m}^{\epsilon}}{\partial t} \mid n_{\gamma}^{2} \frac{\partial \widetilde{u}_{m}^{\epsilon}}{\partial t}\right)_{\mu} d \theta+a_{0, \mu, \gamma}^{\epsilon}\left(t ; \widetilde{u}_{m}^{\epsilon},-\frac{\partial \widetilde{u}_{m}^{\epsilon}}{\partial t}\right)=\int_{(0, m)}\left(f-A_{1} \widetilde{u}_{m}^{\epsilon} \mid-n_{\gamma}^{2} \frac{\partial \widetilde{u}_{m}^{\epsilon}}{\partial t}\right)_{\mu} d \theta,
$$

where

$$
a_{0, \mu, \gamma}^{\epsilon}(t ; u, v)=\epsilon \int_{\widetilde{\mathcal{O}}_{\theta, x}} \frac{\partial u}{\partial \vartheta} \frac{\partial v}{\partial \vartheta} m_{\mu}^{2} n_{\gamma}^{2} d \mathcal{O}+\int_{\widetilde{\mathcal{O}}_{\theta, x}} a_{2}(t) \frac{\partial u}{\partial x} \frac{\partial v}{\partial x} m_{\mu}^{2} n_{\gamma}^{2} d \mathcal{O}
$$

and

$$
A_{1} u=\frac{\partial(b u)}{\partial \vartheta}-a_{1} \frac{\partial u}{\partial x}-a_{0} u
$$

in which case

$$
\begin{gathered}
\int_{(0, m)} n_{\gamma}^{2}\left|\frac{\partial \widetilde{u}_{m}^{\epsilon}}{\partial t}\right|_{\mu}^{2} d \theta-\frac{1}{2} \frac{d}{d t} a_{0, \mu, \gamma}^{\epsilon}\left(t ; \widetilde{u}_{m}^{\epsilon}, \widetilde{u}_{m}^{\epsilon}\right)+\frac{1}{2} \dot{a}_{0, \mu, \gamma}^{\epsilon}\left(t ; \widetilde{u}_{m}^{\epsilon}, \widetilde{u}_{m}^{\epsilon}\right) \\
=\int_{(0, m)} n_{\gamma}^{2}\left(f-A_{1} \widetilde{u}_{m}^{\epsilon},-\frac{\partial \widetilde{u}_{m}^{\epsilon}}{\partial \vartheta}\right)_{\mu} d \theta,
\end{gathered}
$$


where

$$
\dot{a}_{0, \mu, \gamma}^{\epsilon}(\vartheta ; u, v)=\int_{\widetilde{\mathcal{O}}_{\theta, x}} \frac{d a_{2}(t)}{d t} \frac{\partial u}{\partial x} \frac{\partial v}{\partial x} m_{\mu}^{2} n_{\gamma}^{2} d \mathcal{O} .
$$

Integrating the above in time, we have that

$$
\begin{aligned}
& \int_{\widetilde{\mathcal{O}}_{T, \vartheta}} n_{\gamma}^{2}\left|\frac{\partial \widetilde{u}_{m}^{\epsilon}}{\partial t}\right|_{\mu}^{2} d \mathcal{O}+\frac{1}{2} a_{0, \mu, \gamma}^{\epsilon}\left(0 ; \widetilde{u}_{m}^{\epsilon}, \widetilde{u}_{m}^{\epsilon}\right)=\frac{1}{2} a_{0, \mu, \gamma}^{\epsilon}\left(T ; \widetilde{u}_{m}^{\epsilon}, \widetilde{u}_{m}^{\epsilon}\right) \\
& -\frac{1}{2} \int_{0}^{T} \dot{a}_{0}^{\epsilon}\left(t ; \widetilde{u}_{m}^{\epsilon}, \widetilde{u}_{m}^{\epsilon}\right) d t+\int_{\widetilde{\mathcal{O}}_{T, \vartheta}} n_{\gamma}^{2}\left(f-A_{1} \widetilde{u}_{m}^{\epsilon},-\frac{\partial \widetilde{u}_{m}^{\epsilon}}{\partial t}\right)_{\mu} d \mathcal{O} .
\end{aligned}
$$

With (21), we proceed as per Lemma 2 to obtain

$$
\int_{\widetilde{\mathcal{O}}_{T, \vartheta}} n_{\gamma}^{2}\left|\frac{\partial \widetilde{u}_{m}^{\epsilon}}{\partial t}\right|_{\mu}^{2} d \mathcal{O} \leq C
$$

which is valid for all $\widetilde{u}_{m}^{\epsilon}$; passing to the limit, we obtain

$$
\left\|\frac{\partial \widetilde{u}_{m}}{\partial t}\right\|_{\gamma, \mu} \leq C
$$

which holds for all $m$. We determine the estimate in $\vartheta$ analogously.

In the following result, we establish the existence of the solution to (8)-(9) as well as its approximation by the regularization (16)-(19).

Proposition 2. Existence. We suppose (3)-(7), (11), (20), $0<\gamma<c / \bar{b}$ and $f \in L_{\gamma}^{2}\left(\mathcal{O}_{t, \vartheta} ; H_{\mu}\right)$; then, there exists a $u \in W_{\gamma, \mu}$ satisfying (8)-(9). Moreover, the sequence $\left\{\widetilde{u}_{m}\right\}$ converges such that $\widetilde{u}_{m} \rightarrow u$ in $\mathcal{X}_{\gamma, \mu}$ and

$$
\max _{\boldsymbol{t} \in \mathcal{\mathcal { O }}}\left\{\int_{t}^{T} \mid \sqrt{b(\tau, \vartheta)}\left(\widetilde{u}_{m}(\tau, \vartheta)-\left.u(\tau, \vartheta)\right|_{\mu} ^{2} d \tau+\int_{\vartheta}^{\Theta}\left|\widetilde{u}_{m}(t, \theta)-u(t, \theta)\right|_{\mu}^{2} d \theta\right\} \rightarrow 0,\right.
$$

as $m \rightarrow \infty$, where $\mathcal{O}=(0, T) \times(0, \Theta)$, for any $($ fixed $) \Theta>0$.

Proof of Proposition 2. From the estimates (21) and (22), it follows that, possibly after extracting a subsequence, $\tilde{u}_{m} \rightarrow u$ in $\mathcal{X}_{\gamma, \mu}, \partial \tilde{u}_{m} / \partial t \rightarrow \partial u / \partial t$ in $\left.L_{\gamma}^{2}\left(\mathcal{O}, H_{\mu}\right)\right)$ and $\partial \tilde{u}_{m} / \partial \vartheta \rightarrow \partial u / \partial \vartheta$ in $\left.L_{\gamma}^{2}\left(\mathcal{O}, H_{\mu}\right)\right)$, where $u$ satisfies (8)-(9).

In order to show convergence of the regularizations $\widetilde{u}_{m}$, we have from (8) that

$$
\begin{gathered}
-\frac{1}{2} \frac{\partial}{\partial t}|u(\boldsymbol{t})-\widetilde{u}(\boldsymbol{t})|_{\mu}^{2}-\frac{1}{2} \frac{\partial}{\partial \vartheta}|\sqrt{b(\boldsymbol{t})}(u(\boldsymbol{t})-\widetilde{u}(\boldsymbol{t}))|_{\mu}^{2} \\
+a_{\mu}(\boldsymbol{t} ; u(\boldsymbol{t})-\widetilde{u}(\boldsymbol{t}), u(\boldsymbol{t})-\widetilde{u}(\boldsymbol{t}))=(f(\boldsymbol{t}) \mid u(\boldsymbol{t})-\widetilde{u}(\boldsymbol{t}))_{\mu} .
\end{gathered}
$$

Multiplying the above by $n_{\gamma}^{2}$ and applying the Green's formula over $\mathcal{O}$, we obtain

$$
\begin{gathered}
\int_{t}^{T}\left|\sqrt{b(\tau, \vartheta)} u_{n}(\tau, \vartheta)-u(\tau, \vartheta)\right|_{\mu}^{2} d \tau+\int_{\vartheta}^{\Theta}\left|u_{n}(t, \theta)-u(t, \theta)\right|_{\mu}^{2} d \theta \\
+2 \int_{\mathcal{O}} a_{\mu}(\boldsymbol{t} ; u(\boldsymbol{t})-\widetilde{u}(\boldsymbol{t}), u(\boldsymbol{t})-\widetilde{u}(\boldsymbol{t})) d \mathcal{O}=2 \int_{\mathcal{O}}(f(\boldsymbol{t}) \mid u(\boldsymbol{t})-\widetilde{u}(\boldsymbol{t}))_{\mu} d \mathcal{O},
\end{gathered}
$$

and the result follows from (11) and $\tilde{u}_{m} \rightarrow u$ in $\mathcal{X}_{\gamma, \mu}$. 
Let $\left\{w_{1}, w_{2}, \ldots\right\}$ denote a basis in $V_{m}$. We set

$$
\begin{gathered}
u_{m, n}(t, \vartheta, x)=\sum_{k=1}^{n} c_{k n}(t, \vartheta) w_{k}(x), \\
v_{n}(\vartheta, x)=\sum_{k=1}^{n} \alpha_{k n}(\vartheta) w_{k}(x),
\end{gathered}
$$

where $c_{k n}(t, \vartheta) \in L_{2}((0, T) \times(0, m))$ and $\alpha_{k n}(\vartheta) \in L_{2}(0, m)$, such that $\alpha_{k n}(m)=0, v_{n} \in L_{2}\left(0, m ; V_{m}\right)$ and

$$
v_{m, n} \rightarrow v \text { in } L_{2}\left(0, m ; H_{m}\right),
$$

as $n \rightarrow \infty$. The Galerkin equations associated with (16)-(19) are defined

$$
\begin{gathered}
-\sum_{k=1}^{n} \frac{\partial}{\partial t} c_{k n}(\boldsymbol{t})\left(w_{k} \mid w_{j}\right)_{H_{m}}-\sum_{k=1}^{n} \frac{\partial}{\partial \vartheta} c_{k n}(\boldsymbol{t})\left(\sqrt{b(\boldsymbol{t})} w_{k} \mid \sqrt{b(\boldsymbol{t})} w_{j}\right)_{H_{m}} \\
+\sum_{k=1}^{n} c_{k n}(\boldsymbol{t}) a\left(\boldsymbol{t} ; w_{k} \mid w_{j}\right)=\left\langle f(\boldsymbol{t}), w_{j}\right\rangle_{V_{m}} \text { on }(0, T) \times(0, m)
\end{gathered}
$$

for $j=1, \ldots, n$, such that

$$
\begin{gathered}
c_{k n}(T, \vartheta)=\alpha_{k n}(\vartheta) \text { a.e. on }(0, m), \\
c_{k n}(t, m)=0 \text { a.e. on }(0, T),
\end{gathered}
$$

for $k=1, \ldots, n$, where

$$
a(t ; u, v)=\int_{(0, X)} a_{2} \frac{\partial u}{\partial x} \frac{\partial v}{\partial x} d x+\int_{(0, X)} a_{1} \frac{\partial u}{\partial x} v d x+\int_{(0, X)} a_{0} u v d x,
$$

for all $u, v \in V$ and $t \in(0, T) \times(0, m),(u \mid v)_{H_{m}}$ is the inner product on the Hilbert space $H_{m}$, and $\langle f, v\rangle_{V_{m}}$ is the value of the linear functional $f \in V_{m}^{*}$ at $v \in V_{m}$.

We immediately obtain the constructive approximation of (8)-(9)) by the Galerkin procedure (28)-(33) from ([5], Propositions 4 and 5) and Proposition 2.

Proposition 3. Galerkin Approximation. We suppose (3)-(7), (11), (20), $0<\gamma<c / 2 \bar{b}$, and $f \in$ $L_{\gamma}^{2}\left(\mathcal{O}_{t, \vartheta} ; H_{\mu}\right)$. Let $u_{m, m}$ be the $m^{\text {th }}$-Galerkin approximation to $u_{m}$ defined by (28)-(33) and $u$ the solution to (8)-(9), then $u_{m, m} \rightarrow u$ in $\mathcal{X}$ and

$$
\max _{\boldsymbol{t} \in \overline{\mathcal{O}}}\left\{\int_{t}^{T}\left|\sqrt{b(\tau, \vartheta)} u_{m, m}(\tau, \vartheta)-u(\tau, \vartheta)\right|_{\mu}^{2} d \tau+\int_{\vartheta}^{\Theta}\left|u_{m, m}(t, \theta)-u(t, \theta)\right|_{\mu}^{2} d \theta\right\} \rightarrow 0
$$

as $m \rightarrow \infty$, where $\mathcal{O}=(0, T) \times(0, \Theta)$, for any (fixed) $\Theta>0$.

Remark 3. Propositions 2 and 3 likewise hold with $f \in \mathcal{X}_{\gamma, \mu}^{*}$, where we imply the Galerkin approximation per the proof of Lemma 3.

\section{Probabilistic Interpretation}

In order to provide a probabilistic interpretation of the solution to (1)-(2), we make the additional assumptions that

$$
\begin{gathered}
a_{2}, a_{1}, a_{0}, b \in C^{1}(\overline{\widetilde{\mathcal{Q}}}) ; a_{2} \text { bounded, } \\
\frac{\partial^{2} a_{2}}{\partial x^{2}}, \frac{\partial^{2} a_{2}}{\partial x \partial \vartheta}, \frac{\partial^{2} a_{2}}{\partial \vartheta^{2}} \in C^{0}(\overline{\widetilde{\mathcal{Q}}}), \text { bounded, }
\end{gathered}
$$




$$
\begin{gathered}
\frac{\partial a_{0}}{\partial x}, \frac{\partial a_{1}}{\partial x}, \frac{\partial a_{2}}{\partial x}, \frac{\partial b}{\partial x} \text { bounded, } \\
\frac{\partial a_{0}}{\partial \vartheta}, \frac{\partial a_{1}}{\partial \vartheta}, \frac{\partial a_{2}}{\partial \vartheta}, \frac{\partial b}{\partial \vartheta} \text { bounded, } \\
\frac{\partial^{2} a_{2}}{\partial t \partial x}, \frac{\partial^{2} a_{2}}{\partial t \partial \vartheta}, \frac{\partial a_{2}}{\partial x^{2}} \in L_{l o c}^{p}(\widetilde{\mathcal{Q}}), \\
f, \frac{\partial f}{\partial x} \in C^{0}(\overline{\widetilde{\mathcal{Q}}}), \\
|f(t, \vartheta, x)| \leq\left[1+\left(\vartheta^{2}+x^{2}\right)^{m / 2}\right], \\
\left|\frac{\partial f}{\partial x}\right| \leq C\left[1+\left(\vartheta^{2}+x^{2}\right)^{m / 2}\right], \\
\frac{\partial f}{\partial t} \in L_{l o c}^{p}(\widetilde{\mathcal{Q}}) .
\end{gathered}
$$

We likewise suppose the existence of a function

$$
\begin{gathered}
\Psi \in C^{2,1}(\widetilde{\mathcal{Q}}) \cap C^{0}(\overline{\widetilde{\mathcal{Q}}}), \\
\left|\frac{\partial \Psi}{\partial x}\right| \leq C\left[1+\left(\vartheta^{2}+x^{2}\right)^{m / 2}\right],
\end{gathered}
$$

such that

$$
v(\vartheta, x)=\Psi(T, \vartheta, x)
$$

and

$$
g=-\frac{\partial \Psi}{\partial t}-\frac{\partial(b \Psi)}{\partial \vartheta}+A(t, \vartheta) \Psi
$$

satisfies the same assumptions as $f$. From Proposition 2 and Appendix A, we allow that there exists a unique solution $u \in C^{2,1}(\widetilde{\mathcal{Q}}) \cap C^{0}(\overline{\widetilde{\mathcal{Q}}})$ to the problem (1)-(2).

We let $\sigma=\sqrt{2 a_{2}}$ (or $\left.a_{2}=\sigma^{2} / 2\right)$ and define

$$
a(t, \vartheta, x)=\frac{\partial a_{2}}{\partial x}-a_{1}
$$

and

$$
\alpha_{0}=a_{0}-\frac{\partial b}{\partial \vartheta},
$$

in which case $\alpha_{0}>0$ for $\beta$ sufficiently large (cf. (7)). In particular, $\sigma, a$ and $b$ are elements of $C^{1}(\overline{\widetilde{\mathcal{Q}}})$. Moreover, by extending the functions $a, b$, and $\sigma$ outside of $\overline{\mathcal{Q}}$, we may assume that

$$
\begin{aligned}
& \left|\sigma\left(t, \vartheta_{2}, x_{2}\right)-\sigma\left(t, \vartheta_{1}, x_{1}\right)\right|+\left|a\left(t, \vartheta_{2}, x_{2}\right)-a\left(t, \vartheta_{2}, x_{1}\right)\right| \\
& \quad+\left|b\left(t, \vartheta_{2}, x_{2}\right)-b\left(t, \vartheta_{1}, x_{1}\right)\right| \leq K\left(\left|\vartheta_{2}-\vartheta_{1}\right|^{2}+\left|x_{2}-x_{1}\right|^{2}\right)^{1 / 2}
\end{aligned}
$$

as well as

$$
|\sigma| \leq K_{o} \text { and }|a(t, \vartheta, x)|^{2}+|b(t, \vartheta, x)|^{2} \leq K_{1}^{2}\left(1+|\vartheta|^{2}+|x|^{2}\right)
$$

for all $\boldsymbol{t} \in \overline{\widetilde{\mathcal{O}}_{T, \vartheta}}$.

We now seek a probabilistic interpretation of the function $u$ satisfying (1)-(2) by constructing a stochastic differential equation for which the trajectories $(\Theta(t), X(t))$ are the characteristics of $-\partial(b \cdot) / \partial \vartheta+A$. To this end, we take a probability space $(\Omega, \mathcal{A}, P)$, an increasing family of 
sub- $\sigma$-algebras $\mathcal{F}_{t}$ of $\mathcal{A}$, and a $\mathbb{R}$-valued standardized Wiener process $w(t)$, which is an $\mathcal{F}_{t}$ martingale. We can then consider, on an arbitrary finite interval, the stochastic differential equation

$$
\begin{gathered}
d X(t)=a(t, \Theta(t), X(t)) d t+\sigma(t, y) d w(t), \\
d \Theta(t)=b(t, \Theta(t), X(t)) d t \\
X(0)=x \in \mathbb{R} \\
\Theta(0)=\vartheta \in \mathbb{R}_{+},
\end{gathered}
$$

where $x$ and $\vartheta$ are fixed and non-random; the solution of (51)-(54) is unique.

Proposition 4. The assumptions of Proposition 2, as well as (34) through (46); the solution of (1)-(2) is given by

$$
\begin{aligned}
u(t, \vartheta, x) & =\mathbb{E}\left\{\int_{t}^{T} f(s, \Theta(s), X(s)) \exp \left[-\int_{t}^{s} \alpha_{0}(s, \Theta(s), X(\varsigma)) d \varsigma\right] d s\right\} \\
& +\mathbb{E}\left\{v(\Theta(T), X(T)) \exp \left[-\int_{t}^{T} \alpha_{0}(s, \Theta(s), X(s)) d s\right]\right\} .
\end{aligned}
$$

Proof of Proposition 4. The proof relies on the existence and uniqueness of the regular solution to the ultraparabolic terminal value problem (1)-(2). With this exception, the result is standard such that we will provide only a brief exposition, deferring to e.g., ([12], Chapter 2, Theorem 7.4). For the process $(\Theta(t), X(t))$, we have that

$$
\mathbb{E}\left[\left(|\Theta(s)|^{2}+|X(s)|^{2}\right)^{k}\right] \leq C\left[1+\left(|\vartheta|^{2}+|x|^{2}\right)^{k}\right]
$$

for all $s \in[t, T]$ and all $k \in \mathbb{N}$, in which case the right-hand side of (55) is well-defined.

We shall now prove (55) in the case $v=0$. We set

$$
Z(s)=\exp \left\{-\int_{t}^{s}\left[a_{0}(\varsigma, \Theta(\varsigma), X(\varsigma))-\frac{\partial b}{\partial \vartheta}\right] d \varsigma\right\} .
$$

Then, $Z(s)$ satisfies

$$
\frac{d Z(s)}{d s}=\frac{\partial b}{\partial \vartheta}-a_{0}(s, \Theta(s), X(s)) Z(s), Z(t)=1 .
$$

Differentiating the functional $\Psi \cdot Z$, applying Ito's formula to $\Psi$, and integrating from $t$ to $T$, we obtain

$$
\begin{aligned}
\Psi(T, \Theta(T), X(T))=\Psi(t, \vartheta, x) \\
+\int_{t}^{T}\left[\frac{\partial \Psi}{\partial s}+\frac{\partial(b \Psi)}{\partial \vartheta}-A(s, \vartheta) \Psi\right](s, \Theta(s), X(s)) Z(s) d s \\
+\int_{t}^{T} Z(s)\left[\frac{\partial \Psi}{\partial x} \sigma\right](s, \Theta(s), X(s)) d w .
\end{aligned}
$$

From (56) with $k=m$ and the assumptions (44) on the growth of $\partial \Psi / \partial x$, we have that the expectation of the stochastic integral is defined and is equal to zero. We therefore have that

$$
\mathbb{E}\{v(\Theta(T), X(T)) Z(T)\}=\Psi(t, \vartheta, x)-\mathbb{E}\left\{\int_{t}^{T} g(s, \Theta(s), X(s)) Z(s)\right\},
$$


in which case (55) is identical to

$$
u(\boldsymbol{t}, x)-\Psi(t, \vartheta, x)=\mathbb{E}\left\{\int_{t}^{T}[f(s, \Theta(s), X(s))-g(s, \Theta(s), X(s))] Z(s)\right\}
$$

and so the problem reduces to proving (55) with $v=0$, with $f$ replaced by $f-g$, and with $u$ replaced by $u-\Psi$ and a solution to (1)-(2) corresponding to data $f-g$ and 0 .

We therefore assume $v=0$; we prove that

$$
u(t, \vartheta, x)=\mathbb{E}\left\{\int_{t}^{T} f(s, \Theta(s), X(s)) Z(s) d s\right\} .
$$

We start by considering the bounded case. We approximate $f$ by $f_{N M}$ defined by

$$
f_{N M}=\left\{\begin{array}{rll}
N & \text { if } \quad N \leq f \\
f & \text { if } \quad-M \leq f \leq N \\
-M & \text { if } \quad f \leq-M
\end{array}\right.
$$

Since $f_{N M} \in C^{0}(\widetilde{\widetilde{\mathcal{O}}})$ and $\partial f_{N M} / \partial x, \partial f_{N M} / \partial t, \partial f_{N M} / \partial \vartheta \in L_{\text {loc }}^{p}(\widetilde{\mathcal{O}})$, we can uniquely define $u_{N M}$ as the solution of

$$
u_{N M} \in L^{2}\left(\widetilde{\mathcal{O}}_{T, \vartheta} ; H_{\mu}^{1}\right) \cap C^{0}(\widetilde{\widetilde{\mathcal{Q}}}) \cap C^{2,1}(\mathcal{Q})
$$

such that

$$
-\frac{\partial u_{N M}}{\partial t}-\frac{\partial b u_{N M}}{\partial \vartheta}+A(t) u_{N M}=f_{N M}
$$

and

$$
u_{N M}(T, \vartheta, x)=0 .
$$

We note that $u_{M N}$ is bounded. This follows as per ([5], Prop. 2').

We show:

$$
u_{N M}(t, \vartheta, x)=\mathbb{E}\left\{\int_{t}^{T} f_{N M}(s, \Theta(s), X(s)) Z(s) d s\right\} .
$$

To this end, let $\mathcal{O}_{R}=\left\{\xi \in \mathbb{R}^{2}|| \xi \mid \leq R\right\}$ and $\tau_{R}$ be the exit time form $\mathcal{O}_{R}$ of the process $(\Theta(t), X(t))$. We can suppose that the (fixed) initial data $(x, \vartheta)$ of $(51)-(54)$ belongs to $\mathcal{O}_{R}$, for $R$ that is sufficiently large. That is, we have, from the continuity of the process a.s. $\tau_{R} \geq T$ for some $R_{0}(\omega)$ with $R \geq R_{0}(\omega)$, in which case

$$
\text { a.s. } \tau_{R} \wedge T=T \text {, }
$$

for $R \geq R_{0}(\omega)$. As above, with the use of Ito's formula applied to $u_{N M}$ between the instants $t$ and $\tau_{R} \wedge T-\epsilon$, taking $\epsilon \rightarrow 0$, and using the continuity of $u_{N M}$ on $\widetilde{\mathcal{Q}}$, we have

$$
\begin{gathered}
u_{N M}(t, \vartheta, x)=\mathbb{E}\left\{\int_{t}^{\tau_{R} \wedge T} f_{N M}(s, \Theta(s), X(s)) Z(s) d s\right\} \\
+\mathbb{E}\left\{u_{N M}\left(\tau_{R}, \Theta\left(\tau_{R}\right), X\left(\tau_{R}\right)\right) Z\left(\tau_{R}\right) \chi_{T}\left(\tau_{R}\right)\right\},
\end{gathered}
$$

where

$$
\chi_{T}(t)= \begin{cases}1, & \text { if } t<T \\ 0, & \text { if } t \geq T\end{cases}
$$

However, from (63), we have

$$
u_{N M}\left(\tau_{R}, \Theta\left(\tau_{R}\right), X\left(\tau_{R}\right)\right) z\left(\tau_{R}\right) \chi_{T}\left(\tau_{R}\right)=0,
$$


for $R \geq R_{0}(\omega)$, and so

$$
\mathbb{E}\left\{u_{N M}\left(\tau_{R}, \Theta\left(\tau_{R}\right), X\left(\tau_{R}\right)\right) Z\left(\tau_{R}\right) \chi_{T}\left(\tau_{R}\right)\right\} \rightarrow 0 \text { a.s. , }
$$

as $R \rightarrow \infty$. Application of Lesbesque's theorem then provides the result (62).

From the estimates

$$
\left|u_{N M}\right| \leq C\left[1+\left(\vartheta^{2}+x^{2}\right)^{m / 2}\right]
$$

and

$$
\left|\frac{\partial u_{N M}}{\partial x}\right| \leq C\left[1+\left(\vartheta^{2}+x^{2}\right)^{m / 2}\right]
$$

it follows that $u_{N M}$ lies in a bounded subset of $L^{2}\left(\widetilde{\mathcal{O}}_{T, \vartheta} ; H_{\mu}^{1}\right)$ and we obtain (59) by proceeding to the limit successively in $M$ and $N$.

\section{Conclusions}

We have demonstrated the existence and uniqueness of the solution to linear ultraparabolic equations on unbounded domains, both spatial and temporal, as well as the strong convergence of the regularized problem, providing a basis for the subsequent application of a Galerkin approximation. Furthermore, we present a probabilistic interpretation of the solution in terms of the expectation of an associative ultradiffusion process. In practice, the usefulness of this result often stems from the converse formulation; that is, one often wishes to obtain the discounted expectation associated with an ultradiffusion process, e.g., the valuation of an Asian option in mathematical finance (cf. [11]). To this end, the regularity assumptions of Section 3 are necessary for the existence of the solution to the ultradiffusion process (3.5). With respect to a simple regular transformation, the associated ultraparabolic problem maintains the approximation solvability of Section 2, for which efficient and general numerical procedures are readily available (cf. [13-15]).

Funding: This research received no external funding.

Conflicts of Interest: The authors declare no conflicts of interest.

\section{Appendix A. Regularity}

There exist two approaches to obtaining regularity; for parabolic differential equations of the second order, we note that Wloka [16] has provided regularity theorems based on raising the differentiability assumptions of the data while Ladyženskaja et al. [17] have taken the approach of increasing the $p$-power summability of the data. The results stated below follow the latter approach, the demonstration of which lies outside the scope of this manuscript. As regularity theorems are local, they do not require assumptions on the boundary or the boundedness of the domain .

We let $\mathcal{W}^{1,2, p}(\widetilde{\mathcal{Q}})$ denote the space of functions $u$ such that

$$
u, \frac{\partial u}{\partial t}, \frac{\partial u}{\partial \vartheta}, \frac{\partial u}{\partial x}, \frac{\partial^{2} u}{\partial x^{2}} \in L^{p}(\widetilde{\mathcal{Q}}),
$$

for $1 \leq p \leq \infty$. Here, the " 1 " refers to the order of temporal derivatives, and " 2 " refers to the number of spatial derivatives. If $p=2$, we write $\mathcal{W}^{1,2}(\widetilde{\mathcal{Q}})$, which we equip with the natural Banachand Hilbert-space norm. We denote by $\mathcal{W}_{\text {loc }}^{1,2, p}(\widetilde{\mathcal{Q}})$ the space of functions $u$ such that, for all test functions $\varphi \in \mathcal{D}(\widetilde{\mathcal{Q}})$, the set of infinitely differentiable functions with compact support in $\widetilde{\mathcal{Q}}$, we have $\varphi u \in \mathcal{W}_{\mathrm{loc}}^{1,2, p}(\widetilde{\mathcal{Q}})$.

We suppose that

$$
a_{2}, a_{1}, a_{0}, b \in C^{1}(\widetilde{\mathcal{Q}}) .
$$


Moreover, for $v \in L_{\text {loc }}^{p}(\widetilde{\mathcal{Q}})$, we denote by $L v$ the following distribution on $\widetilde{\mathcal{Q}}$ :

$$
\langle L v, \psi\rangle=\int_{\widetilde{\mathcal{Q}}} v\left[\frac{\partial \psi}{\partial t}+\frac{\partial(b \psi)}{\partial \vartheta}-\frac{\partial}{\partial x}\left(a_{2} \frac{\partial \psi}{\partial x}\right)-\frac{\partial}{\partial x}\left(a_{1} \psi\right)+a_{0} \psi\right] d \widetilde{\mathcal{Q}},
$$

for all $\psi \in \mathcal{D}(\widetilde{\mathcal{Q}})$.

Proposition A1. Local Regularity. The assumptions of Proposition 2, as well as (65). Let $u \in L_{\mathrm{loc}}^{p}(\widetilde{\mathcal{Q}})$ be such that

$$
L u=-\frac{\partial u}{\partial t}-\frac{\partial(b u)}{\partial \vartheta}+A(t, \vartheta) u=f \in L_{\mathrm{loc}}^{p}(\widetilde{\mathcal{Q}}),
$$

then $u \in \mathcal{W}_{\text {loc }}^{1,2, p}(\widetilde{\mathcal{Q}})$, for $p>1$.

Proof of Proposition A1. The case for $p=2$ follows with a slight modification from ([12], Chapter 2, Theorem 5.5).

In order to obtain results on the boundary, we set

$$
\widetilde{f}= \begin{cases}f, & \text { on }(0, T), \\ 0, & \text { on }(-T, 0) \text { and }(T, 2 T),\end{cases}
$$

and extend the operator $A(t, \vartheta)$ in such a way that it is defined over $(-T, 2 T)$, all the while retaining the properties of the coefficients. Finally, we consider the solution of $\widetilde{u}$ of

$$
-\frac{\partial \widetilde{u}}{\partial t}-\frac{\partial(b \widetilde{u})}{\partial \vartheta}+A(t, \vartheta) \widetilde{u}=\widetilde{f} \text { a.e. on } \widetilde{\mathcal{Q}}
$$

subject to the terminal condition

$$
\widetilde{u}(2 T, \vartheta, x)=0 \text { a.e. on } \widetilde{\mathcal{O}}_{\vartheta, x} .
$$

Corollary A1. The assumptions of Proposition A1; we have

$$
u \in C^{0}(\overline{\widetilde{\mathcal{Q}}})
$$

for $p>2$.

Proof of Corollary A1. From Proposition A1 applied to $\widetilde{u}$ in (66)-(67), we obtain $\widetilde{u} \in \mathcal{W}_{\text {loc }}^{1,2, p}(\widetilde{\mathcal{Q}})$ from which we derive the result.

Increased smoothness of the data may then be translated into smoothness of the solution.

Proposition A2. The assumptions of Proposition A1, as well as

$$
\begin{gathered}
\frac{\partial^{2} a_{2}}{\partial t \partial x}, \frac{\partial^{2} a_{2}}{\partial \vartheta \partial x}, \frac{\partial^{2} a_{2}}{\partial x^{2}} \in L_{\mathrm{loc}}^{p}(\widetilde{\mathcal{Q}}), \\
f, \frac{\partial f}{\partial t}, \frac{\partial f}{\partial \vartheta}, \frac{\partial f}{\partial x} \in L_{\mathrm{loc}}^{p}(\widetilde{\mathcal{Q}}), \\
u \in L_{\mathrm{loc}}^{p}(\widetilde{\mathcal{Q}}),
\end{gathered}
$$

and $L u=f$, then

$$
u \in \mathcal{W}_{\mathrm{loc}}^{1,3, p}(\widetilde{\mathcal{Q}})
$$


and

$$
\frac{\partial u}{\partial t}, \frac{\partial u}{\partial \vartheta} \in \mathcal{W}_{\text {loc }}^{1,2, p}(\widetilde{\mathcal{Q}})
$$

In particular, $u \in C^{1,2}(\widetilde{\mathcal{Q}})$.

Proof of Proposition A2. We consider the differential quotients technique per Section 4 of [5] such that, if $f \in \mathcal{W}_{\text {loc }}^{1,1, p}(\widetilde{\mathcal{Q}})$, then $u \in \mathcal{W}_{\text {loc }}^{1,3, p}(\widetilde{\mathcal{Q}})$.

Finally, the key regularity result is:

Proposition A3. The assumptions of Proposition A2. If $f \in L^{p}(\widetilde{\mathcal{Q}})$ and $v=0$, then the unique solution to (1)-(2) also satisfies $u \in L_{\mathrm{loc}}^{p}(\widetilde{\mathcal{Q}})$.

\section{Appendix B. Localization}

In order to highlight the temporal nature of the variable $v$ in the probabilistic framework, we examine formally the localization of the ultradiffusion process (51)-(54) and its relation to (1)-(2). To this end, we freeze the drift and volitility in a neighborhood of $t=0$ and consider the ultradiffusion process

$$
\begin{array}{r}
d X(t)=a d t+\sigma d w(t), \\
d \Theta(t)=b d t, \\
X(0)=x \in \mathbb{R}, \\
\Theta(0)=\vartheta \in \mathbb{R}_{+},
\end{array}
$$

where $x$ and $\vartheta$ are again fixed and non-random. It follows then that the solution to ultraparabolic infinite horizon/ terminal boundary value problem

$$
\begin{gathered}
-\frac{\partial u}{\partial t}-\frac{\partial(b u)}{\partial \vartheta}+A u=0 \text { a.e. on } \widetilde{\mathcal{Q}}, \\
u(T, \vartheta, x)=v(\vartheta, x) \text { a.e. on } \widetilde{\mathcal{O}}_{\vartheta, x}
\end{gathered}
$$

may be characterized as

$$
u(t, \vartheta, x)=\mathbb{E}\left\{v(\Theta(T), X(T)) \exp \left[-a_{0}(T-t)\right]\right\} .
$$

In particular, we consider the temporal characteristic transformation $t=t(\tau)$ and $\vartheta=\vartheta(\tau)$ such that

$$
\begin{gathered}
\frac{d t(\tau)}{d \tau}=1 ; t(0)=0, \\
\frac{d \vartheta(\tau)}{d \tau}=b ; \vartheta(0)=\vartheta_{0},
\end{gathered}
$$

where $\vartheta_{0}>0$, in which case $t(\tau)=\tau$ and $\vartheta(\tau)=b \tau+\vartheta_{0}$ or, more simply, $t=\tau$ and $\vartheta(t)=b t+\vartheta_{0}$. Note then that, along the characteristic line $(t(\tau), \vartheta(\tau))$, the Formulations (70)-(76) may be restated in terms of the characteristic parameterized diffusion

$$
\begin{gathered}
d \mathcal{X}\left(\tau ; \vartheta_{0}\right)=a d \tau+\sigma d \omega(\tau), \\
\mathcal{X}\left(0 ; \vartheta_{0}\right)=x \in \mathbb{R},
\end{gathered}
$$

such that the solution $v\left(\tau, x ; \vartheta_{0}\right)=u(t(\tau), \vartheta(\tau), x)$ to the characteristic parameterized parabolic terminal value problem 


$$
\begin{aligned}
& -\frac{\partial v}{\partial \tau}+A v=0 \text { a.e. on }(0, T) \times \mathbb{R}, \\
& v\left(T, x ; \vartheta_{0}\right)=v\left(\vartheta_{0}, x\right) \text { a.e. on } \widetilde{\mathcal{O}}_{\vartheta, x}
\end{aligned}
$$

satisfies

$$
v(\tau, x)=\mathbb{E}^{\prime}\left\{v(\mathcal{X}(T)) \exp \left[-a_{0}(T-\tau)\right]\right\} .
$$

The temporal nature of $\vartheta$ then follows from the characteristic problem (77)-(83). Due to (77)-(78), the vector $(1, b)$ (resp. $b$ ) is known as the velocity (resp. speed) of propagation.

\section{References}

1. Piskunov, N.S. Problémes Limits pour les Équations du Type Elliptic-Parabolique. Mat. Sb. 1940, 7, 385-424.

2. Lions, J.L. Sur Certaines Équations aux Dérivativées Partielles, à Coefficients Opérateurs non Bornés. J. Anal. Math. 1958, 6, 333-355. [CrossRef]

3. Il'in, A.M. On a Class of Ultraparabolic Equations. Soviet Math. Dokl. 1964, 5, 1673-1676.

4. Vladimirov, V.S.; Drožžinov, J.N. Generalized Cauchy Problem for an Ultraparabolic Equation. Math. USSR Izv. $1967,1,1285-1303$.

5. Marcozzi, M.D. Well-Posedness of Linear Ultraparabolic Equations on Bounded Domains. J. Evol. Equ. 2018, 18, 75-104. [CrossRef]

6. Kolmogorov, A.N. Zur Theorie der Stetigen Zufalligen Progresse. Math. Ann. 1933, 108, 149-160. [CrossRef]

7. Kolmogorov, A.N. Zufällige Bewegungen. Ann. Math. 1934, 35, 116-117.

8. Uhlenbeck, G.E.; Ornstein, L.S. On the Theory of the Brownian Motion. Phys. Rev. 1930, 36, 823-841. [CrossRef]

9. Chandrasekhar, S. Stochastic Problems in Physics and Astronomy. In Selected Papers on Noise and Stochastic Processes; Wax, N., Ed.; Dover: New York, NY, USA, 2013; Reprint.

10. Marshak, R.E. Theory of the Slowing Down of Neutrons by Elastic Collisions with Atomic Nuclei. Rev. Mod. Phys. 1947, 19, 185-238. [CrossRef]

11. Hull, J.C. Options, Futures, and Other Derivatives, 10th ed.; Pearson: New York, NY, USA, 2017.

12. Bensoussan, A.; Lions, J.L. Applications of Variational Inequalities in Stochastic Control; North Holland: Amsterdam, The Netherlands, 1982.

13. Akrivis, G.M.; Crouzeix, M.; Thomee, V. Numerical methods for ultraparabolic equations. Calcolo 1996, 31, 179-190. [CrossRef]

14. Marcozzi, M.D. On the valuation of Asian options by variational methods. SIAM J. Sci. Comput. 2003, 24, 1124-1140. [CrossRef]

15. Marcozzi, M.D. Extrapolation discontinuous Galerkin method for ultraparabolic equations. J. Comput. Appl. Math. 2009, 224, 679-687. [CrossRef]

16. Wloka, J. Partial Differential Equations; Cambridge University Press: Cambridge, UK, 1987.

17. Ladyženskaja, O.A.; Solonnikov, V.A.; Ural'ceva, N.N. Linear and Quasilinear Equations of Parabolic Type; American Mathematical Society: Providence, RI, USA, 1967; Volume 23 of Translations of Mathematical Monographs.

(C) 2018 by the author. Licensee MDPI, Basel, Switzerland. This article is an open access article distributed under the terms and conditions of the Creative Commons Attribution (CC BY) license (http://creativecommons.org/licenses/by/4.0/). 